\title{
WNN Nonlinear Modeling Method Based on Heuristic RS Attribute Reduction Algorithm and Its Application
}

\author{
Gan Xusheng ${ }^{1, a}$, Qu Hong ${ }^{2, b}$, Han Jun ${ }^{2, c}$ \\ ${ }^{1 .}$ XiJing College, Xi’an, Shaanxi, 710123, China; \\ 2. Air Traffic Control and Navigation College, Air Force Engineering University, Xi’an, Shaanxi, 710051, \\ China; \\ aganxusheng123@163.com; bqh3231@163.com; cxbs421@yahoo.com.cn
}

Keywords: Wavelet Neural Network; Rough Set; Attribute Reduction; Nonlinear Modeling

\begin{abstract}
Compared with the traditional neural network, Wavelet Neural Network (WNN) has many advantages, but it cannot achieve the expected modeling effect when dealing with the nonlinear modeling problem with large input dimension. In order to solve this problem, a WNN modeling method based on Rough Set (RS) attribute reduction is proposed. In this method, a heuristic attribute reduction RS algorithm is used to reduce the input variables in advance, and then the WNN model is established on this basis. The experiment result on the aerodynamic modeling of the aircraft shows that the proposed method is effective and feasible, so as to provide a good choice for the nonlinear modeling of complex systems.
\end{abstract}

\section{Introduction}

Wavelet Neural Network (WNN) is a special form of neural network. It not only inherits the advantages of adaptive learning, parallel processing, information distribution and storage of neural networks, but also overcomes the problems of local minima and slow convergence [1]. The disadvantage is that the input information space dimension cannot be simplified. When the input information space dimension is large, the network is not only complex in structure, but also need a long training time. In the analysis of complex databases, the input information has not only large dimension but also noise interferences, simply using WNN cannot achieve the desired results.

The emergence of Rough Set (RS) theory brings an opportunity to solve this problem. Through analyzing the inherent relationship between data information, RS can not only remove redundant information, but also simplify the space dimension of input information, that is, it can find the inherent rules of data information. But it also exist the shortcoming in fault tolerance, generalization ability and processing of qualitative information. Based on above analysis, RS is integrated with WNN to construct a new modeling method, which can reduce the complexity of WNN structure, shorten the training time and improve the generalization performance of WNN in theory.

To process complex problems effectively, a heuristic RS attribute reduction recursive algorithm is introduced to reduce the redundant attributes. Simulation verifies its effectiveness.

\section{Wavelet Neural Network}

WNN proposed by Zhang Q. $H$. is a new neural network, as shown in Fig. 1, where $x_{i}(i=1,2, \cdots, I)$ is the network input, $y_{k}(k=1,2, \cdots, K)$ is the network output, $w_{j i}$ is the connection weight from input layer to hidden layer; $w_{k j}$ is the connection weight from hidden layer to output layer. $I, J, K$ are the nodes number of input layer, hidden layer and output layer respectively. $\psi(\cdot)$ is the wavelet basis function in hidden layer. 


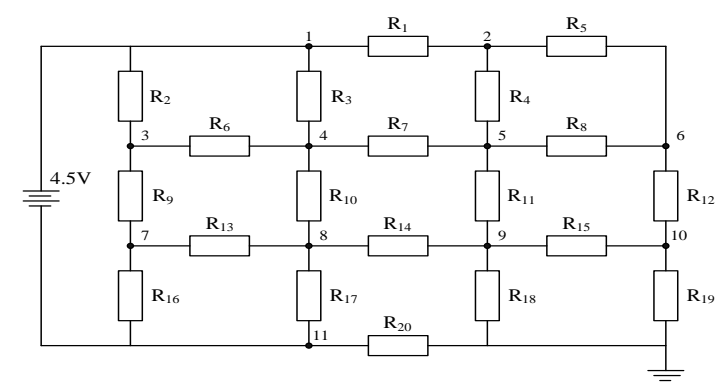

Fig. 1. Structure of WNN

\section{A Heuristic Attribute Reduction Algorithm of RS}

Attribute reduction is one of the core problems in RS theory research. A decision system may have many relative reductions, it is always desirable to get the reduction with the least attribute, i.e. the minimum reduction, and it has been proved that finding the minimum reduction is the NP-hard problem. This problem is usually based on the heuristic search method. By adding heuristic information to the algorithm, the search space of the problem is reduced to obtain easily the optimal solution or approximate optimal solution. In determining the optimal decision attributes before WNN modeling, on the basis of hierarchical structure and approximation precision concept, a heuristic attribute reduction recursive algorithm is introduced to effectively solve complex problems [2][3].

In the decision information system $(U, C \cup D, V, f), U$ is the object set, and $C$ is the condition attribute set, $D$ is the decision attribute set, $V=\bigcup_{r \in R} V_{r}\left(V_{r}\right.$ is the value range of attribute $\left.r \in R\right)$; $f$ can be thought of as the function between $U$ and $R\left(f: U \times R \rightarrow V, f(x, r) \in V_{r}\right)$. Suppose that $X$ is a subset of $U$. For each $X$, the indiscernibility relation $B(B \subseteq A)$.

For the condition attributes, if the decision value of the objects in indiscernibility relation set is the same, then the decision system is compatible; otherwise, it is incompatible. In the attribute reduction algorithm, the equivalence relation of all condition attributes firstly need to be determined in compatible decision information system, then the upper and lower approximation sets with respect to decision value are calculated for each equivalence relation. The heuristic parameter $\xi$ can be obtained by

$$
\xi=\frac{\left(\left|B^{*}(X)\right|-\left|B_{*}(X)\right|\right)}{|U|}
$$

where $B_{*}(X)$ is the lower approximation set, $B^{*}(X)$ is the upper approximation set. The attribute with the smallest $\xi$ is selected. For given universe of discourse, the lower approximate value can be used as the basis for pruning

$$
U=U-P_{\text {red }}\{d\}
$$

where $\operatorname{POS}_{\text {red }}\{d\}$ is the object set corresponding to the decision attribute $d$ that the condition attributes of reduction set red can be classified into $U$. Repeat the process and select the attribute with the smallest $\xi$. Each time the remaining attributes are combined with the attributes in the reduction set red to form a new equivalence relation. The algorithm is recursive and its calculation is terminated until the universe of discourse given is empty.

The calculation steps of recursive attribute reduction algorithm are as follows:

1 . The reduction set red can be initialized as $\varnothing$ (empty set), $C$ is all condition attributes.

2. $\xi$ of each condition attribute in $C$ is calculated by the formula (1).

3. All $\xi$ are sorted in descending order, and the attribute with the smallest $\xi$ is selected. If 
two attributes have the same $\xi$, the attribute with the least attribute value can be selected. If $\xi$ is the same and the attribute value is the same, an attribute can be selected at random.

4. The selected attribute $a_{i}$ is put into red and reduced from $C$, conducting the formula (2).

5. If $U$ is $\varnothing$, go to step 7; otherwise, go to the next step.

6 . The condition attributes in red are combined with the remaining condition attributes in $C$, and repeat steps 2 5.

7. The final reduction set red is obtained, and the reduction is terminated.

The process of above attribute reduction algorithm can be illustrated by the decision table as shown in Table 1. $C=\left\{c_{1}, c_{2}, c_{3}, c_{4}\right\}$ is the condition attribute set, $d$ is the decision attribute.

Table 1 Decision Table

\begin{tabular}{llllll}
\hline$U$ & $C_{1}$ & $c_{2}$ & $c_{3}$ & $c_{4}$ & $d$ \\
\hline 1 & 1 & 1 & 1 & 3 & 2 \\
2 & 2 & 1 & 1 & 1 & 1 \\
3 & 1 & 2 & 2 & 2 & 2 \\
4 & 1 & 1 & 3 & 2 & 2 \\
5 & 1 & 1 & 1 & 1 & 1 \\
6 & 2 & 2 & 2 & 2 & 1 \\
7 & 2 & 2 & 2 & 3 & 2 \\
8 & 1 & 1 & 3 & 1 & 1 \\
9 & 1 & 2 & 3 & 2 & 2 \\
10 & 1 & 2 & 2 & 1 & 2 \\
\hline
\end{tabular}

For each condition attribute in Table 1, the formula (1) is used to calculate the heuristic parameter $\xi$, obtaining the results in Table 2 .

Table 2 First Round Indiscernibility Relation Set

\begin{tabular}{cccccc}
\hline Decision attribute & $c_{1}$ & $c_{2}$ & $c_{3}$ & $c_{4}$ \\
\hline \multirow{2}{*}{$d=1$} & Lower & $\varnothing$ & $\varnothing$ & $\varnothing$ & $\varnothing$ \\
& Upper & $U$ & $U$ & $U$ & $\begin{array}{c}2,3,4,5, \\
6,8,9,10\end{array}$ \\
& Lower & $\varnothing$ & $\varnothing$ & $\varnothing$ & 1,7 \\
$d=2$ & & & & & \\
& Upper & $U$ & $U$ & $U$ & $U$ \\
\hline & $\xi$ & 2 & 2 & 2 & $8 / 5$ \\
\hline
\end{tabular}

Example: considering the condition attribute $C_{4}$

The decision attribute $d=1$ :

$$
\begin{aligned}
& B^{*}(X)=\{2,3,4,5,6,8,9,10\}, \quad\left|B^{*}(X)\right|=8 ; \\
& B_{*}(X)=\varnothing,\left|B_{*}(X)\right|=0 ;|U|=10 \\
& \xi_{1}=\frac{\left(\left|B^{*}(X)\right|-\left|B_{*}(X)\right|\right)}{|U|}=\frac{8-0}{10}=4 / 5
\end{aligned}
$$

The decision attribute $d=2$ : 


$$
\begin{aligned}
& B^{*}(X)=U,\left|B^{*}(X)\right|=10 ; \\
& B_{*}(X)=\{1,7\},\left|B_{*}(X)\right|=2 ;|U|=10 \\
& \xi_{2}=\frac{\left(\left|B^{*}(X)\right|-\left|B_{*}(X)\right|\right)}{|U|}=\frac{10-2}{10}=4 / 5 \\
& \xi=\xi_{1}+\xi_{2}=4 / 5+4 / 5=8 / 5
\end{aligned}
$$

Through the same process, the heuristic parameter $\xi$ of $c_{1}, c_{2}, c_{3}$ can also be calculated. It can be seen from Table 2 that $\xi$ of $c_{4}$ is the smallest in $c_{1}, c_{2}, c_{3}, c_{4}$, then the attribute $c_{4}$ can be placed in the reduction set red, that is

$$
\begin{aligned}
& \text { red } \leftarrow\left\{c_{4}\right\}, \text { red }=\left\{c_{4}\right\} \\
& C \leftarrow C-\text { red }, C=\left\{c_{1}, c_{2}, c_{3}\right\}
\end{aligned}
$$

Use the formula (2) to prune the domain, and the reduced domain is

$$
\begin{aligned}
& P_{\text {red }}\{d\}=\{1,7\} \\
& U \leftarrow U-\operatorname{POS}_{\text {red }}\{d\}=\{2,3,4,5,6,8,9,10\}
\end{aligned}
$$

Subsequently, the processing procedure is repeated using the combined attributes, as shown in

\begin{tabular}{|c|c|c|c|c|}
\hline \multicolumn{2}{|c|}{ Decision attribute } & \multirow{2}{*}{$\frac{C_{4}, C_{1}}{2,6}$} & \multirow{2}{*}{$\frac{C_{4}, C_{2}}{2,5,8}$} & \multirow{2}{*}{$\frac{C_{4}, C_{3}}{2,5,8}$} \\
\hline & Lower & & & \\
\hline$d=1$ & Upper & $\begin{array}{c}2,6,5 \\
8,10\end{array}$ & $\begin{array}{l}2,5,8 \\
3,6,9\end{array}$ & $2,5,8,3,6$ \\
\hline \multirow{2}{*}{$d=2$} & Lower & $3,4,9$ & 4,10 & $4,9,10$ \\
\hline & Upper & $\begin{array}{c}3,4,9,5, \\
8,10\end{array}$ & $\begin{array}{c}3,4,6 \\
9,10\end{array}$ & $\begin{array}{c}3,4,6,9 \\
10\end{array}$ \\
\hline \multicolumn{2}{|c|}{$\xi$} & $3 / 4$ & $3 / 4$ & $1 / 2$ \\
\hline
\end{tabular}
Table 3 and Table 4. Because $\xi$ of $c_{4}$ is the smallest in Table 2, then it can get

$$
\begin{aligned}
& \text { red } \leftarrow \text { red } \bigcup\left\{c_{3}\right\}, \quad \text { red }=\left\{c_{4}, c_{3}\right\} \\
& C \leftarrow C-\text { red }, \quad C=\left\{c_{1}, c_{2}\right\} \\
& U \leftarrow U-P O S_{\text {red }}\{d\}=\{3,6\}
\end{aligned}
$$

Because $\xi$ of $c_{1}$ is the smallest in Table 3, it can get

$$
\begin{aligned}
& \text { red } \leftarrow \text { red } \bigcup\left\{c_{1}\right\}, \text { red }=\left\{c_{4}, c_{3}, c_{1}\right\} \\
& C \leftarrow C-\text { red }=\left\{c_{2}\right\}
\end{aligned}
$$

This moment $U=\varnothing$, that is, according to the condition attribute $c_{4}, c_{3}, c_{1}$, the equivalence relation ind $(d)$ can be distinguished. Accordingly, the reduced attribute subset $r e d=\left\{c_{4}, c_{3}, c_{1}\right\}$.

Table3 Second Round Indiscernibility Relation Set 
Table 4 Third Round Indiscernibility Relation Set

\begin{tabular}{|c|c|c|c|}
\hline \multicolumn{2}{|c|}{ Decision attribute } & \multirow{2}{*}{$\frac{C_{4}, C_{3}, C_{1}}{6}$} & \multirow{2}{*}{$\frac{C_{4}, C_{3}, C_{2}}{\varnothing}$} \\
\hline \multirow{3}{*}{$d=1$} & Lower & & \\
\hline & & & \\
\hline & Upper & 6 & 3,6 \\
\hline \multirow{3}{*}{$d=2$} & Lower & 3 & $\varnothing$ \\
\hline & & & \\
\hline & Upper & 3 & 3,6 \\
\hline \multicolumn{2}{|c|}{$\xi$} & 0 & 2 \\
\hline
\end{tabular}

\section{Realization of RS-WNN}

The basic idea of integration modeling based on RS and WNN: firstly, analyze the sample data, and form an initial information table according to the known knowledge, and then the resolution function method is used to conduct the relative reduction for conditional attributes, the minimum condition attribute set and the kernel of the decision table are obtained by removing the redundant condition attribute. The union set of the minimum condition attribute set is taken as the input of WNN to realize the final decision classification. The specific steps are as follows:

1. Initial decision table formation. In initial sample data collected, some parameters are repeated, while others are missing. When forming a complete initial information table, these repetitive parameters must be deleted, and the necessary characteristic parameters will be supplemented.

2. Decision table formation. Using the condition attributes and decision attributes value to form a two-dimensional table, each row describing an object, each column corresponding to an attribute of the object.

3. Attribute reduction. Using the heuristic attribute reduction method introduced, the decision table is reduced and the minimum condition attribute set is obtained.

4. Model establishing. Use the minimum condition attribute set as input and the decision attribute as output to train the WNN model.

5. Results Analysis: The WNN model was tested using the test samples and the test results were output.

\section{Experiment Simulation}

Aerodynamic modeling of quasi-steady stall is a more complex nonlinear modeling problem. The WNN model which can accurately reflect the aerodynamic characteristics of the quasi-steady stall for aircraft ATTAS is established without reference to any stall hysteresis physical phenomena. The used flight data is measured from ATTAS flight in quasi-steady stall conditions [4]. The longitudinal aerodynamic model of quasi-steady stall for ATTAS can be expressed as

$$
\left[C_{D}, C_{L}, C_{m}\right]=f\left(\delta_{e}, \delta_{a}, \delta_{r}, p, q, r, \alpha, \dot{\alpha}, \beta, V, \rho, F l, F r, M a\right)
$$

In fact, the longitudinal aerodynamic modeling of quasi-steady stall using RS-WNN in the formula (3) is such a process that WNN with inputs $\delta_{e}, \delta_{a}, \delta_{r}, p, q, r, \alpha, \dot{\alpha}, \beta, V, \rho, F l, F r, M a$ and outputs $C_{D}, C_{L}, \quad C_{m}$ is trained to obtain the longitudinal aerodynamic model that can accurately describe the dynamic characteristics of the quasi-steady stall for ATTAS. $p, q, r$ is the roll, pitch and yaw angular rate, $\alpha, \beta$ is the angle of attack and sideslip angle, $V$ is the true velocity, $\delta_{e}, \delta_{a}, \delta_{r}$ are the elevator, aileron and rudder angle, $\rho$ is the atmospheric density, $F l, F r$ are the thrust of left and right engine respectively. $C_{L}, C_{D}$ is the lift and drag force coefficient, $C_{m}$ is the pitching moment coefficient. Then the formula (3) is reduced by RS to obtain $\alpha, q, V, \delta_{e}$, and on the basis, the 
WNN aerodynamic model is established.

Fig. 2 gives the prediction results of the RS-WNN longitudinal aerodynamic model of quasi-steady stall. The training convergence curve of RS-WNN model is shown in Fig. 3. It can be seen from experimental results that the built model can accurately describe the complex aerodynamic phenomenon, and can reflect the dynamic characteristics of quasi-steady stall process.
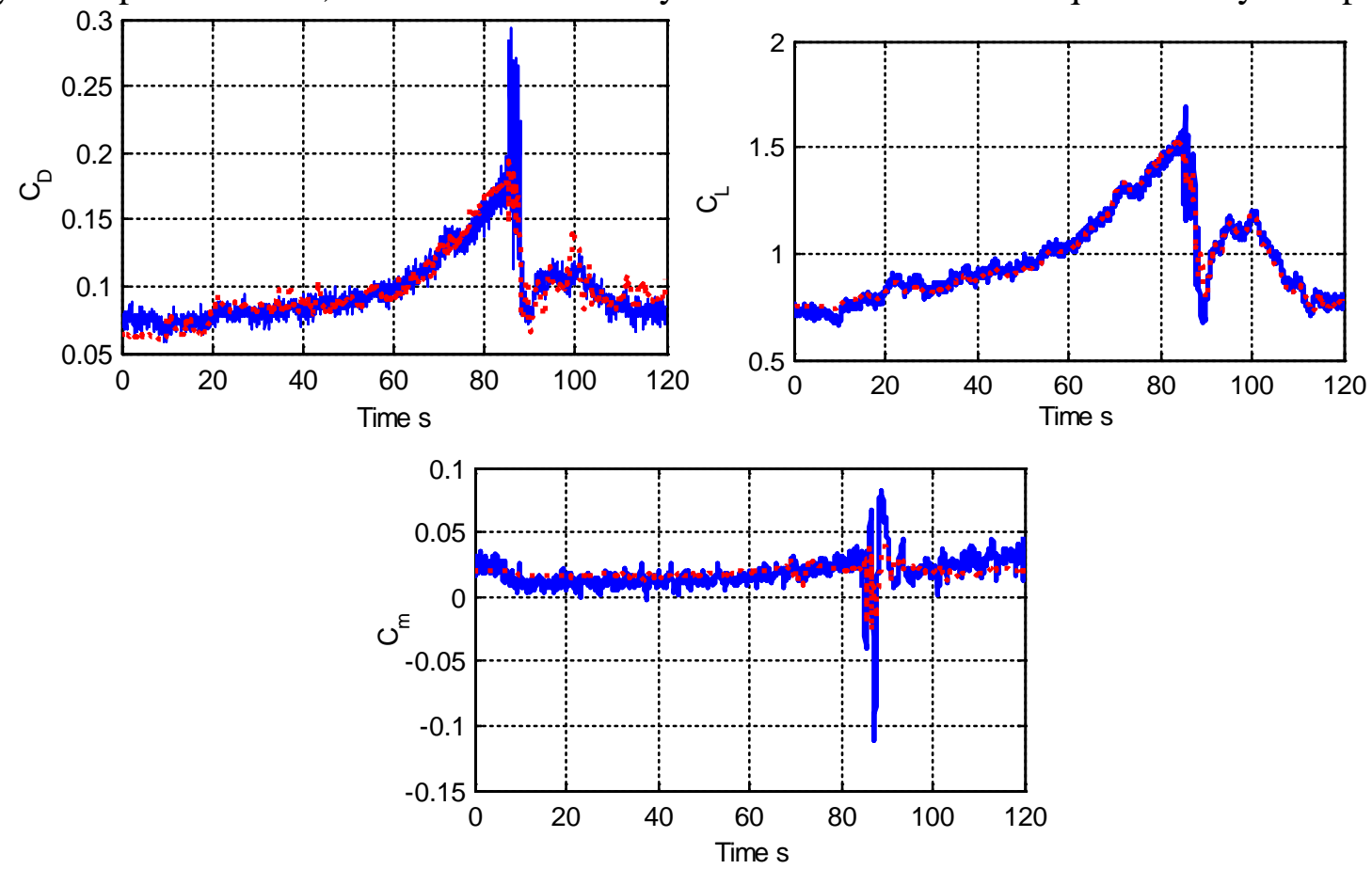

Fig. 2. Prediction results of longitudinal aerodynamic RS-WNN model for ATTAS quasi-stall ( - flight derived, --- model estimated )

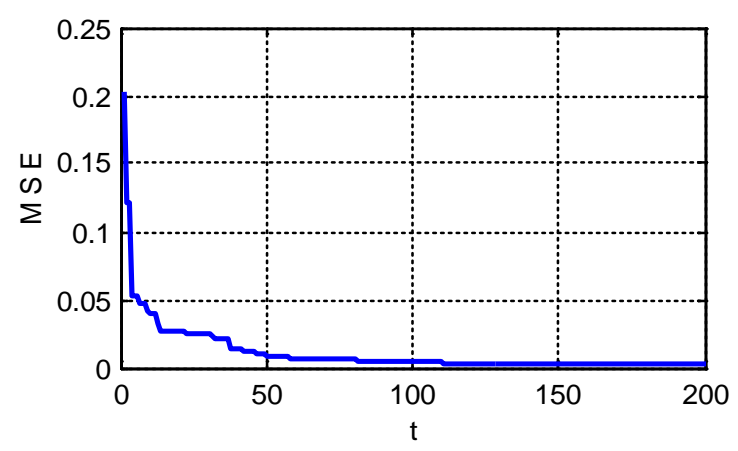

Fig. 3. Convergence curve of RS-WNN model training

\section{Conclusion}

To improve the modeling performance of WNN, a WNN modeling method based on RS is proposed. In the method, a heuristic RS attribute reduction recursive algorithm is introduced to remove the redundant variables in advance before WNN modeling, and then the reduced variables is used to train the model of WNN. The experiment shows that RS-WNN method can solve the problem on nonlinear system modeling.

\section{References}

[1] Q. H. Zhang, A. Benveniste, "Wavelet network," IEEE Trans- actions on Neural Networks, vol. 3, No. 6, 1992, pp. 889-898.

[2] B. B. Qu, Y. S. Lu, "Rough set-based algorithm for attribute reduction,” Journal of Huazhong University of Science and Technology, vol. 33, No. 8, 2005, pp. 30-33. 
[3] B. F. Wu, Q. Li, W. Z. Song, "Inductive learning approach to knowledge representation system based on rough set theory” Control and Decision, vol. 14, No. 3, 1999, pp. 206-211.

[4] X. S. Gan, J. S. Duanmu, Y. B. Meng, W. Cong, "Aerodynamic Modeling from Flight Data Based on WNN Optimized by Particle Swarm," Acta Aeronautica et Astronautica Sinica, vol. 33, No. 7, 2012, pp. 1209-1217. 